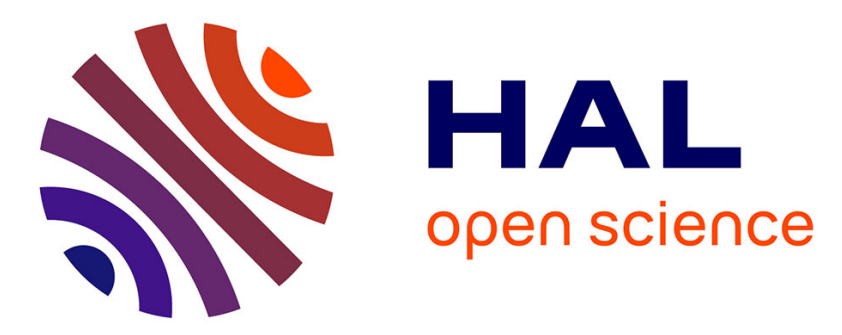

\title{
Estimation of polydispersity in aggregating red blood cells by quantitative ultrasound backscatter analysis
}

Romain de Monchy, Julien Rouyer, François Destrempes, Boris Chayer, Guy Cloutier, Emilie Franceschini

\section{- To cite this version:}

Romain de Monchy, Julien Rouyer, François Destrempes, Boris Chayer, Guy Cloutier, et al.. Estimation of polydispersity in aggregating red blood cells by quantitative ultrasound backscatter analysis. Journal of the Acoustical Society of America, 2018, 143 (4), pp.2207-2216. hal-02016704

\section{HAL Id: hal-02016704 https://hal.science/hal-02016704}

Submitted on 12 Feb 2019

HAL is a multi-disciplinary open access archive for the deposit and dissemination of scientific research documents, whether they are published or not. The documents may come from teaching and research institutions in France or abroad, or from public or private research centers.
L'archive ouverte pluridisciplinaire HAL, est destinée au dépôt et à la diffusion de documents scientifiques de niveau recherche, publiés ou non, émanant des établissements d'enseignement et de recherche français ou étrangers, des laboratoires publics ou privés. 


\title{
Estimation of polydispersity in aggregating red blood cells by quantitative
}

\section{ultrasound backscatter analysis}

\author{
Romain de Monchy, ${ }^{1}$ Julien Rouyer, ${ }^{1}$ François Destrempes, ${ }^{2}$ Boris Chayer, ${ }^{2}$ Guy Cloutier,,${ }^{2, a)}$
} Emilie Franceschini ${ }^{1, b)}$

${ }^{1}$ Laboratoire de Mécanique et d'Acoustique, Aix-Marseille Université, CNRS UPR 7051, Centrale Marseille, 4 impasse Nikola TESLA, CS 40006, 13453 Marseille cedex 13, France

${ }^{2}$ Laboratory of Biorheology and Medical Ultrasonics, University of Montreal Hospital Research Centre (CRCHUM), 900 St-Denis (suite R11.720), Montreal, Quebec, Canada, H2X $0 \mathrm{~A} 9$

a) Also at: Department of Radiology, Radio-Oncology and Nuclear Medicine, and Institute of Biomedical Engineering, University of Montreal, Montreal, Quebec, H3T IJ4, Canada

b) E-mail: franceschini@lma.cnrs-mrs.fr 


\begin{abstract}
Quantitative ultrasound techniques based on the backscatter coefficient (BSC) have been commonly used to characterize red blood cell (RBC) aggregation. Specifically, a scattering model is fitted to measured BSC and estimated parameters can provide a meaningful description of the RBC aggregates' structure (i.e., aggregate size and compactness). In most cases, scattering models assumed monodisperse RBC aggregates. This study proposes the Effective Medium Theory combined with the polydisperse Structure Factor Model (EMTSFM) to incorporate the polydispersity of aggregate size. From the measured BSC, this model allows estimating three structural parameters: the mean radius of the aggregate size distribution, the width of the distribution, and the compactness of the aggregates. Two successive experiments were conducted: a first experiment on blood sheared in a Couette flow device coupled with an ultrasonic probe, and a second experiment, on the same blood sample, sheared in a plane-plane rheometer coupled to a light microscope. Results demonstrated that the polydisperse EMTSFM provided the best fit to the BSC data when compared to the classical monodisperse models for the higher levels of aggregation at hematocrits between 10-40\%. Fitting the polydisperse model yielded aggregate size distributions that were consistent with direct light microscope observations at low hematocrits.
\end{abstract}


de Monchy et al., JASA, p. 1

\section{Introduction}

Quantitative ultrasound (QUS) techniques providing insight into tissue microstructure may be based on spectral analysis of signals backscattered from biological tissues. QUS methods used to determine red blood cell (RBC) aggregate structures may rely on theoretical scattering models in order to fit a theoretical BackScatter Coefficient (BSC) to experimentally measured BSC. The BSC is defined as the power backscattered by a unit volume of scatterers per unit incident intensity per unit solid angle. Two scattering theories, called the Structure Factor Size Estimator (SFSE) and the Effective Medium Theory combined with the Structure Factor Model (EMTSFM), have been developed for practical assessment of RBC structural features. ${ }^{1 ; 2 ; 3}$ The SFSE scattering theory approximates the structure factor with its second-order Taylor expansion. ${ }^{1}$ The SFSE estimates two physical parameters describing the microstructure of $\mathrm{RBC}$ aggregates: the packing factor, which increases with erythrocyte clustering, and the average aggregate isotropic diameter. However, experiments with porcine blood ${ }^{1}$ and three-dimensional (3D) numerical simulations of $\mathrm{RBC}$ aggregates ${ }^{4 ; 5}$ recently revealed that these two structural parameters are correlated, thus reducing the BSC parameterization to one structural index. The SFSE is therefore empirical and only allows assessing one feature of RBC aggregation, i.e. its mean fractal size. This is the reason why the EMTSFM was recently proposed. ${ }^{3 ; 5}$ The EMTSFM assumes that aggregates of RBCs can be treated as individual homogeneous 
de Monchy et al., JASA, p. 2

scatterers, which have effective properties determined by the acoustical characteristics and concentration of RBCs within aggregates. The approximation of RBC aggregates as homogeneous effective particles is combined with the structure factor to model acoustic wave interferences in concentrated blood medium. The EMTSFM allows characterizing the aggregate radius, and for the first time in QUS, the compactness of RBC aggregates. Based on 3D computer simulations of monodisperse aggregates, the EMTSFM was demonstrated to be more suitable than the former scattering theories (i.e., the SFSE and the classical Spherical Gaussian Model) for characterizing RBC aggregation. ${ }^{5}$

Both scattering models SFSE and EMTSFM assume that all RBCs are aggregated in blood, and that aggregates are identical with sphere-shaped structures. Therefore, the BSC behavior obtained from the monodisperse $\mathrm{EMTSFM}^{3}$ or numerical simulations ${ }^{4 ; 5}$ show pronounced frequency peaks. However, in experimental conditions, ${ }^{1 ; 2}$ the BSC behavior turned out to be smoother and peaks were less pronounced. The reason behind this discrepancy might be that blood presents a variable size of aggregates, and since the location of BSC peaks is expected to be different for different aggregate sizes, a relatively smoother BSC curve as a function of frequency might occur. Moreover, optical observations of flowing RBC aggregates at a low hematocrit showed that the aggregates are polydisperse in size. ${ }^{6 ; 7}$

In the field of spectral-based QUS techniques, the local monodisperse 
approximation ${ }^{8 ; 9}$ or the polydisperse structure function ${ }^{10 ; 11}$ were experimentally studied to consider polydisperse scatterers in concentrated medium. Preliminary experimental results on aggregating blood at a low hematocrit of $20 \%$ suggest that taking into account polydispersity can help better understand BSC from aggregating RBCs. ${ }^{9}$ Nonetheless, this work was limited by using the local monodisperse approximation, which is valid only in the case of moderate polydispersity. ${ }^{12}$ Moreover, the benefits of polydisperse modeling was not evaluated against classical monodisperse modeling. Therefore, the present work combines the Effective Medium Theory (EMT) with the polydisperse structure function, and compares the capacities of the polydisperse EMTSFM and the monodisperse models (i.e., the SFSE and the monodisperse EMTSFM) to characterize the aggregate structure in the context of in vitro experiments. Two successive experiments were conducted: a first experiment on blood sheared in a Couette flow device coupled with an ultrasonic probe at hematocrits of 10, 20 and 40\%, and a second experiment, on the same blood sample, sheared in a plane-plane rheometer coupled to a light microscope. The optical measurements were limited to low hematocrits of 10 and $20 \%$ because RBCs are opaque to light, which prevents optical measurements at physiological hematocrit of $40 \%$. The aggregate size distributions estimated by ultrasound and by optical microscopic image segmentation were compared on the same blood samples. This allowed evaluating the performance of the ultrasonic technique using optical detection of aggregate boundaries as 
de Monchy et al., JASA, p. 4

the gold standard measure of aggregate structure.

\section{Ultrasound backscattering theory}

The incident wavelength $\lambda$ is assumed to be larger than the RBC size. Consequently, the biconcave RBC shape can be approximated by a sphere of radius $a$ having an equivalent volume $V_{\mathrm{c}}=\frac{4}{3} \pi a^{3}$ (typically, $V_{\mathrm{c}} \approx 87 \mu \mathrm{m}^{3}$ and $a \approx 2.75 \mu \mathrm{m}$ for human RBCs, whereas $V_{\mathrm{c}} \approx 61 \mu \mathrm{m}^{3}$ and $a \approx 2.45 \mu \mathrm{m}$ for porcine RBCs). RBCs are described in terms of their mass density $\rho_{c}$ and compressibility $\kappa_{c}$, and their surrounding medium (i.e., the plasma) is characterized by its mass density $\rho_{0}$ and compressibility $\kappa_{0}$. We introduce the parameters $\gamma_{\kappa}=\left(\kappa_{c}-\kappa_{0}\right) / \kappa_{0}$ and $\gamma_{\rho}=\left(\rho_{c}-\rho_{0}\right) / \rho_{c}$ corresponding to the fractional variations in compressibility and mass density, respectively. It is also assumed that the mean distance between the red blood cells is not larger than the wavelength and that the aggregates of RBCs are spherical as this can occur in pathological cases. Indeed, the structures of RBC aggregates have a tendency to form clumps in pathological human blood, such as in diabetes mellitus, ${ }^{14 ; 15}$ whereas aggregates of RBCs form "rouleaux" in normal blood (i.e., anisotropic structures).

\section{A. Effective Medium Theory combined with polydisperse Structure Factor}

\section{Model}

\section{Effective Medium Theory for one single RBC aggregate}


de Monchy et al., JASA, p. 5

The Effective Medium Theory (EMT) detailed in Ref. (13) for modeling one aggregate of RBCs is briefly recalled as follows. The EMT model assumes that an aggregate of cells can be treated as an effective homogeneous spherical scatterer of radius $r_{\mathrm{ag}}$. As previously studied by Morse and Ingard,${ }^{16}$ the differential backscattering cross-section $\sigma_{\text {ag }}$ of an aggregate can be decomposed into two components:

$$
\sigma_{\mathrm{ag}}(k)=\sigma_{\mathrm{ag}, \mathrm{coh}}(k)+\sigma_{\mathrm{ag}, \mathrm{inc}}(k),
$$

where the coherent component $\sigma_{\mathrm{ag}, \mathrm{coh}}$ corresponds to the average wave emerging from the effective scatterer, and the incoherent component $\sigma_{\mathrm{ag}, \text { inc }}$ describes the fluctuation of the scattering wave around its average within the effective scatterer.

Using Born and far-field approximations, the backscattering amplitude from a single RBC aggregate $\Phi_{\text {ag }}$ can be expressed as (see Eq. (5) in Ref. (13))

$$
\Phi_{\mathrm{ag}}(k)=\frac{k^{2}\left(\gamma_{\kappa}-\gamma_{\rho}\right)}{4 \pi} V_{\mathrm{c}} F_{0}(k ; a) \sum_{j=1}^{N} e^{2 i k \mathbf{n}_{0} \cdot \mathbf{r}_{j}},
$$

where $k$ is the wavenumber, $\mathbf{n}_{0}$ is the incident wave direction, $\mathbf{r}_{j}$ is the position in three dimensional space of the $j^{\text {th }} \mathrm{RBC}$ (among the $N$ RBCs inside an aggregate) and $F_{0}(k ; a)^{2}=F(k ; a)$ is the spherical form factor of a RBC expressed as ${ }^{17}$

$$
F_{0}(k ; a)=\frac{3(\sin (2 k a)-2 k a \cos (2 k a))}{(2 k a)^{3}} .
$$

By assuming that the RBCs are grouped into spherical aggregates, of radius $r_{\text {ag }}$ and of compactness $\phi_{i}=N V_{c} / V_{\mathrm{ag}}$ (where $V_{\mathrm{ag}}=\frac{4}{3} \pi r_{\mathrm{ag}}^{3}$ is the aggregate volume), the coherent 
component of the differential backscattering cross-section is written as (see Eq. (11) in Ref. (13)):

$$
\begin{aligned}
\sigma_{\mathrm{ag}, \mathrm{coh}}(k) & =\left|\left\langle\Phi_{\mathrm{ag}}(k)\right\rangle\right|^{2} \\
& \approx \frac{k^{4}\left(\gamma_{\kappa, \mathrm{ag}}-\gamma_{\rho, \mathrm{ag}}\right)^{2}}{16 \pi^{2}} V_{\mathrm{ag}}^{2} F\left(k ; r_{\mathrm{ag}}\right),
\end{aligned}
$$

where $\gamma_{\kappa, \mathrm{ag}}=\left(\kappa_{\mathrm{ag}}-\kappa_{0}\right) / \kappa_{0}$ and $\gamma_{\rho, \mathrm{ag}}=\left(\rho_{\mathrm{ag}}-\rho_{0}\right) / \rho_{\mathrm{ag}}$ are the fractional variations in compressibility and in mass density of the effective scatterer, with $\rho_{\text {ag }}$ and $\kappa_{\text {ag }}$ defined as: ${ }^{18}$

$$
\begin{aligned}
& \rho_{\mathrm{ag}}=\phi_{i} \rho_{c}+\left(1-\phi_{i}\right) \rho_{0} \\
& \kappa_{\mathrm{ag}}=\left[\phi_{i} / \kappa_{c}+\left(1-\phi_{i}\right) / \kappa_{0}\right]^{-1} .
\end{aligned}
$$

Note that the scatterer radius used in the spherical form factor of Eq. (4) was directly related to $r_{\text {ag }}$ because the RBCs' positions inside an aggregate are unknown in experimental conditions.

Inside an aggregate, each sphere of radius $a$ produces a scattered wave, with density $\rho_{c}$ and compressibility $\kappa_{c}$, in a surrounding medium of density $\rho_{\mathrm{ag}}$ and compressibility $\kappa_{\mathrm{ag}}$ throughout the aggregate. Therefore, the wavenumber inside the aggregate is modified as ${ }^{16}$ $k_{\mathrm{ag}}=k \sqrt{\rho_{\mathrm{ag}} \kappa_{\mathrm{ag}}} / \sqrt{\rho_{0} \kappa_{0}}$. The incoherent component of the differential backscattering cross-section of the effective scatterer is then modeled as (see Eq. (15) in Ref. (13)):

$$
\begin{aligned}
\sigma_{\mathrm{ag}, \text { inc }}(k) & =\left\langle\left|\Phi_{\mathrm{ag}}\left(k_{\mathrm{ag}}\right)-\left\langle\Phi_{\mathrm{ag}}\left(k_{\mathrm{ag}}\right)\right\rangle\right|^{2}\right\rangle \\
& \approx \frac{k_{\mathrm{ag}}^{4}\left(\gamma_{\kappa}-\gamma_{\rho}\right)^{2}}{16 \pi^{2}} V_{\mathrm{ag}} V_{\mathrm{c}} \phi_{i} F\left(k_{\mathrm{ag}} ; a\right) S\left(k_{\mathrm{ag}} ; a, \phi_{i}\right),
\end{aligned}
$$

where $S$ is the structure factor for a collection of randomly distributed identical RBCs of 
de Monchy et al., JASA, p. 7

radius $a$ and of concentration $\phi_{i}$ within an aggregate (analytically calculated as established by Wertheim $\left.{ }^{19}\right)$.

Previous computer simulations demonstrated that the theoretical differential backscattering cross-section $\sigma_{\text {ag }}$ of an aggregate (given by Eqs. (1), (4) and (6)) allows to approximate satisfactorily the simulated $\sigma_{\text {ag }}$ within a large frequency bandwidth ranging from 1 to $100 \mathrm{MHz}$ for spherical aggregate size up to $r_{\mathrm{ag}} / a=9$ (see Fig. 5 in Ref. (13)). The relative errors between simulated and theoretical $\sigma_{\text {ag }}$ were shown to be less than $15 \%$ for a product of the wavenumber times the mean aggregate radius $k r_{\mathrm{ag}} \leq 2$.

\section{Polydisperse and monodisperse structure functions}

The EMT combined with the polydisperse structure function (named in the sequel the polydisperse EMTSFM) assumes that RBC aggregates are polydisperse in size and that the distribution $p$ of the aggregates' radius follows a gamma probability density function $(\mathrm{PDF}):^{10 ; 20}$

$$
p_{\left(\bar{r}_{\mathrm{ag}}, z\right)}\left(r_{\mathrm{ag}}\right)=\frac{r_{\mathrm{ag}}^{z}}{\Gamma(z+1)}\left(\frac{z+1}{\bar{r}_{\mathrm{ag}}}\right)^{z+1} e^{-(z+1) r_{\mathrm{ag}} / \bar{r}_{\mathrm{ag}}},
$$

where $\bar{r}_{\text {ag }}$ is the mean aggregate radius and $z$ is the gamma width factor which is inversely related to the width of the distribution (a large value of $z$ corresponds to a narrow size distribution). In the sequel of the paper, $p$ will be assumed to be gamma distributed and the subscript $\left(\bar{r}_{\mathrm{ag}}, z\right)$ will be omitted.

Assuming that the aggregate compactness $\phi_{i}$ is identical for all aggregates (except in 
the case of a single RBC having an aggregate compactness of 1 ), the BSC can be written as the sum of an incoherent scattering contribution that sums up the intensities of the backscattered waves of each effective scatterer independently and of a coherent scattering contribution originating from the spatial correlation in effective scatterer positions:

$$
B S C_{\text {EMTpoly }}(k)=B S C_{\text {inc }}(k)+B S C_{\text {coh }}(k),
$$

with

$$
\begin{aligned}
B S C_{\mathrm{inc}}(k)= & m_{\mathrm{ag}} \int_{0}^{\infty}\left\langle\left|\Phi_{\mathrm{ag}}\left(k, r_{\mathrm{ag}}\right)\right|^{2}\right\rangle p\left(r_{\mathrm{ag}}\right) \mathrm{d} r_{\mathrm{ag}}, \\
B S C_{\mathrm{coh}}(k)= & m_{\mathrm{ag}} \iint_{0}^{\infty}\left\langle\Phi_{\mathrm{ag}}\left(k, r_{\mathrm{ag} 1}\right)\right\rangle\left\langle\Phi_{\mathrm{ag}}\left(k, r_{\mathrm{ag} 2}\right)\right\rangle \\
& H_{12}(k) p\left(r_{\mathrm{ag} 1}\right) p\left(r_{\mathrm{ag} 2}\right) \mathrm{d} r_{\mathrm{ag} 1} \mathrm{~d} r_{\mathrm{ag} 2},
\end{aligned}
$$

where $H_{12}$ is the partial structure function between the effective scatterers of radii $r_{\mathrm{ag} 1}$ and $r_{\mathrm{ag} 2}$, and $m_{\mathrm{ag}}$ is the number density of effective spheres defined as:

$$
m_{\mathrm{ag}}=\frac{\phi_{\mathrm{ag}}}{(4 / 3) \pi \int_{a}^{\infty} p\left(r_{\mathrm{ag}}\right) r_{\mathrm{ag}}^{3} d r_{\mathrm{ag}}} .
$$

The volume fraction of an effective scatterer $\phi_{\text {ag }}$ is computed as follows:

$$
\phi_{\mathrm{ag}}=\psi \phi+(1-\psi) \phi / \phi_{i},
$$

where $\phi$ denotes the hematocrit (i.e., the volume fraction of RBCs) and $\psi$ is the ratio between the number of disaggregated RBCs (for which the aggregate compactness is $\left.\phi_{i}=100 \%\right)$ and the total number of RBCs. 
de Monchy et al., JASA, p. 9

When all RBCs are disaggregated, one has $\psi=1$ and $\phi_{\mathrm{ag}}=\phi$. When all RBCs are aggregated with a compactness $\phi_{i}$, one has $\psi=0$ and $\phi_{\mathrm{ag}}=\phi / \phi_{i}$. Note that, when $r_{\mathrm{ag}} / a=1$ (disaggregated case), the differential backscattering cross-section is that of a single RBC:

$$
\sigma_{b}(k)=\frac{k^{4}\left(\gamma_{\kappa}-\gamma_{\rho}\right)^{2}}{16 \pi^{2}} V_{\mathrm{c}}^{2} F(k ; a) .
$$

In the proposed study, the term $B S C_{\text {coh }}$ was computed using the structure function (denoted $S F$ ) of the effective particle defined as the ratio between the total BSC and the incoherent $B S C_{\text {inc: }}{ }^{10 ; 20}$

$$
B S C_{\mathrm{coh}}(k)=B S C_{\mathrm{inc}}(k)(S F(k)-1)
$$

where the expression of the structure function $S F$ is given in Appendix B of Ref. (10).

In the case of a monodisperse size distribution (i.e., an ensemble of identical aggregates of radius $r_{\mathrm{ag}}$ ), the monodisperse EMTSFM allows expressing the BSC as follows: ${ }^{5}$

$$
B S C_{\mathrm{EMTmono}}(k)=m_{\mathrm{ag}} \sigma_{\mathrm{ag}}(k) S_{\mathrm{ag}}(k),
$$

where $\sigma_{\mathrm{ag}}$ is the differential backscattering cross-section of an aggregate computed using Eqs. (1), (4) and (6), and $S_{\mathrm{ag}}$ corresponds to the structure factor of a collection of $N_{\mathrm{ag}}$ identical effective particles of radius $r_{\text {ag }}$ randomly distributed (analytically calculated as established by Wertheim ${ }^{19}$ ). 
de Monchy et al., JASA, p. 10

B. Structure Factor Size Estimator (SFSE) The structure factor model (SFM)

sums up the acoustic contributions from individual RBCs and models their interaction by a statistical mechanics structure factor $S$. The SFSE developed by Yu and Cloutier ${ }^{1}$ approximates the SFM with a second-order Taylor expansion of the structure factor in $k$. By assuming identical RBCs and identical isotropic aggregates, the SFSE model approximates the BSC as follows:

$$
B S C_{\mathrm{SFSE}}(k)=m \sigma_{b}(k)\left(W-\frac{12}{5}(k a D)^{2}\right)
$$

where $m$ is the number of RBCs per unit volume $\left(m=\phi / V_{s}\right), \sigma_{b}$ is the differential backscattering cross-section of a single RBC computed using Eq. (12), $W$ is the low-frequency limit of the structure factor $\left(\left.S(k)\right|_{k \rightarrow 0}\right)$ called the packing factor and $D$ is the mean aggregate diameter expressed in number of RBCs. ${ }^{1}$

\section{Methods}

A. Blood samples preparation Fresh porcine whole blood was obtained from a local slaughter house and anticoagulated with $3 \mathrm{~g} / \mathrm{L}$ of ethylene diamine tetra acetic acid (EDTA). The whole blood was centrifuged and the plasma and buffy coat were removed. The RBCs were then washed three times in a saline solution.

Three aggregating blood samples, denoted S10, S20 and S40, were prepared with an hematocrit of 10, 20 and 40\%, respectively. The sample preparation was performed as 
de Monchy et al., JASA, p. 11

follows. The RBCs were resuspended in a mixture containing $75 \%$ of saline solution and $25 \%$ of Optiprep ${ }^{\mathrm{TM}}$ (iodixanol solution, Progen Biotechnik), and $1.5 \mathrm{~g} / \mathrm{L}$ of dextran 500 kDa (Sigma Aldrich, Saint Quentin Fallavier, France) was then dissolved in the reconstituted blood. The Optiprep ${ }^{\mathrm{TM}}$ allows adjusting the density of the suspending medium at around $1080 \mathrm{~kg} / \mathrm{m}^{3}$ and to prevent sedimentation of RBCs. The dose and molecular weight of dextran allowed producing highly aggregated blood ${ }^{21}$ with a tendency to form quasi-spherical aggregates and to mimic pathological blood.

A disaggregated blood sample, denoted H4, was prepared with an hematocrit of $4 \%$. The H4 sample consisted of RBCs resuspended in a mixture containing $75 \%$ of saline solution and $25 \%$ of Optiprep ${ }^{\mathrm{TM}}$ to prevent the aggregation process in the absence of macromolecules (dextran or plasma proteins).

\section{B. Ultrasound measurements in the Couette flow device US measurements were}

performed in a Couette device to produce a linear blood velocity profile at a given rotational speed to obtain a constant shear rate. The system consists of a rotating inner cylinder with a diameter of $160 \mathrm{~mm}$ surrounded by a fixed concentric cylinder of diameter $164 \mathrm{~mm}$ (see Fig. 1 in Ref. (23)). A $60 \mathrm{~mL}$ blood sample was sheared in the annular space between both coaxial cylinders of length $e=2 \mathrm{~mm}$. Ultrasonic data were acquired using a high frequency system (Vevo 770, Visualsonics Inc, Toronto, Canada). Two probes, RMV 716 and RMV 703, were used in B-mode imaging. For the RMV 716 and the RMV 703 
de Monchy et al., JASA, p. 12

probes, the oscillating single-element focused circular transducers had center frequencies of 16 and $30 \mathrm{MHz}$, focuses of 17.5 and $10 \mathrm{~mm}$ and f-numbers of 2.1 and 2.5, respectively. Raw $\mathrm{RF}$ data were digitized at a sampling frequency of $250 \mathrm{MHz}$ (12-bit precision) using a high-speed acquisition card (Gagescope, model CS12501, Montreal, Canada). The selected probe was mounted in the side wall of the fixed outer cylinder and was positioned to have its focal zone at the center of the gap between both cylinders. To ensure ultrasonic coupling, the hole within the outer stationary cylinder (containing the probe) was filled with a liquid agar-agar gel based mixture in order to match the curvature of the cylinder and to avoid any flow disturbance.

All US measurements were made at room temperature. Prior to each measurement, the blood sample (S10, S20 or S40) was sheared at $300 \mathrm{~s}^{-1}$ during $30 \mathrm{~s}$ to disrupt RBC aggregates. The shear rate was then fixed to values of $3,6,11,22,45$ and $90 \mathrm{~s}^{-1}$ for $60 \mathrm{~s}$ until a steady equilibrium in the state of aggregation was reached. The smallest shear rate produces the highest level of aggregation, and vice versa. For each shear rate, twelve B-mode images were successively constructed from acquired RF echoes for a total period of analysis of $90 \mathrm{~s}$. For 100 scan lines in the center of B-mode images, echoes were selected within a rectangular window of axial length corresponding to twelve wavelengths. The power spectra of the 100 backscattered echoes were then averaged to provide $\overline{P_{\text {meas }}}$. The blood sample preparation and the ultrasonic measurement protocol was repeated twice 
de Monchy et al., JASA, p. 13

with different porcine bloods for hematocrits of 10, 20 and $40 \%$.

C. Measured backscatter coefficients and attenuation First, attenuation of blood samples was estimated using a standard substitution method. ${ }^{22}$ Experiments were conducted with a focused transducer with a center frequency of $20 \mathrm{MHz}$ (with -10 dB bandwidth of 11-35 MHz in water), a focus of $25 \mathrm{~mm}$, and an f-number of 2.1. The rotating inner cylinder was used as a reflector. The transducer was used in emission/reception and the transducer focus was positioned at the interface between the blood sample under investigation and the rotating inner cylinder. The substitution method consists in comparing the spectra of the echoes reflected by the inner cylinder both with blood and with water inserted in the ultrasound path and sheared in the Couette flow device. The attenuation was found to depend linearly on frequency, within the 11-35 MHz frequency bandwidth. For the highest level of aggregation (i.e., for the shear rate of $3 \mathrm{~s}^{-1}$ ), averaged attenuation coefficients were $0.09,0.38$ and $0.81 \mathrm{~dB} / \mathrm{cm} / \mathrm{MHz}$ for the samples S10, S20 and S40, respectively. For the lowest level of aggregation (i.e., for the shear rate of $90 \mathrm{~s}^{-1}$ ), averaged attenuation coefficients were $0.03,0.11$ and $0.20 \mathrm{~dB} / \mathrm{cm} / \mathrm{MHz}$ for the samples S10, S20 and S40, respectively. For the disaggregated sample H4, averaged attenuation coefficient was $0.02 \mathrm{~dB} / \mathrm{cm} / \mathrm{MHz}$.

The measured BSC values reported in this study were computed using the reference phantom technique. ${ }^{24 ; 25}$ The reference scattering medium makes it possible to compensate 
de Monchy et al., JASA, p. 14

the measured backscattered power spectrum $\overline{P_{\text {meas }}}$ for the electromechanical system response and the depth-dependent diffraction and focusing effects caused by the ultrasound beam. The reference scattering medium was a disaggregated blood sample ${ }^{25}$ at a low hematocrit of $4 \%$ (i.e., Rayleigh scatterers) sheared at $22 \mathrm{~s}^{-1}$ in the Couette flow device. Echoes from the reference scattering medium were acquired and windowed as with the aggregating blood sample, and their power spectra were averaged to obtain $\overline{P_{\text {ref }}}$. The measured BSC was thus computed as follows

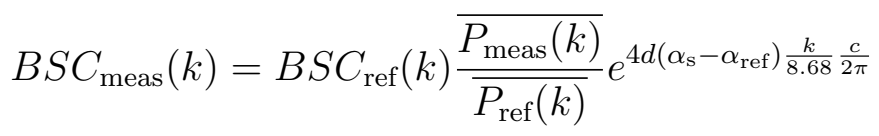

where $d$ is the distance from the interface agar/blood to the center of the windowed analysis region, $\alpha_{\mathrm{s}}$ and $\alpha_{\text {ref }}$ are the predetermined attenuation coefficients (in $\mathrm{dB} / \mathrm{mm} / \mathrm{MHz}$ ) of the aggregating blood sample and of the reference disaggregated blood sample. The coefficient 8.68 expresses unit conversion from decibels to neper. The $B S C_{\text {ref }}$ corresponds to the BSC of the disaggregated blood sample estimated by using the expression of the Perkus-Yevick packing factor for spheres $W_{\mathrm{PY}}$ as follows: ${ }^{27 ; 28}$

$$
B S C_{\mathrm{ref}}(k)=m \sigma_{b}(k) W_{\mathrm{PY}}=m \sigma_{b}(k) \frac{(1-\phi)^{4}}{(1+2 \phi)^{2}} .
$$

The Percus-Yevick packing factor theory was previously experimentally assessed on disaggregated blood sample using a substitution method with a planar reflector (see Fig. 1 in Ref. (1)). This procedure yielded a $B S C_{\text {ref }}$ for each probe. The two $B S C_{\text {meas }}$ were then 
de Monchy et al., JASA, p. 15

combined to yield a single $B S C_{\text {meas }}$ over the combined bandwidth of the two transducers (i.e., $11-41 \mathrm{MHz})$.

D. QUS parameters estimation The three tested models, namely, the monodisperse SFSE and EMTSFM, and the polydisperse EMTSFM, assume that the hematocrit $\phi$, the $\mathrm{RBC}$ radius $a$ and the acoustical properties of plasma and RBCs are prior knowledge. A theoretical model was fitted to the experimental BSC to estimate QUS parameters (i.e. $W^{*}$ and $D^{*}$ using the SFSE, $r_{\mathrm{ag}}^{*}$ and $\phi_{i}^{*}$ using the monodisperse EMTSFM, and $\bar{r}_{\mathrm{ag}}^{*}, z^{*}$ and $\phi_{i}^{*}$ using the polydisperse EMTSFM) by minimizing the cost function $\mathcal{F}$ defined as:

$$
\mathcal{F}=\sum_{i}\left\|\frac{B S C_{\text {meas }}\left(k_{i}\right)-B S C_{\text {theo }}\left(k_{i}\right)}{B S C_{\text {meas }}\left(k_{i}\right)}\right\|^{2},
$$

where $B S C_{\text {theo }}$ is given by Eq. (15) for the SFSE, by Eq. (14) for the monodisperse EMTSFM and by Eq. (8) for the polydisperse EMTSFM. In the case of the SFSE, a quadratric function of the two variables $W$ and $D^{2}$ is obtained, and by calculating its critical points, a system of two linear equations with two unknowns can be solved. In the case of the monodisperse EMTSFM, ${ }^{5}$ the routine fminsearch of MATLAB (The Mathworks Inc., Natick, MA), i.e., the Nelder-Mead simplex method, was employed to minimize the cost function $\mathcal{F}$; whereas in the case of the polydisperse EMTSFM, we used the routine fmincon of MATLAB, with the constraints $0 \leq \phi_{i} \leq 1$ and $p_{\left(\bar{r}_{\mathrm{ag}}, z\right)}\left(r_{\mathrm{ag}}<a\right)=0$ (i.e., the aggregate radius should be larger than the RBC radius a). For both monodisperse and 
de Monchy et al., JASA, p. 16

polydisperse EMTSFM, ten random initializations were used in our implementation. In the sequel of the paper, the standard deviation of the aggregate radius distribution $\sigma_{\mathrm{ag}}^{*}=\bar{r}_{\mathrm{ag}}^{*} / \sqrt{z^{*}+1}$ will be reported instead of the gamma width factor $z^{*}$.

For each studied shear rate and sample, the goodness of fit of the three models to the experimentally measured $\mathrm{BSC}_{\text {meas }}$ was assessed by the goodness-of-fit statistic, $R^{2}$, given by: ${ }^{29}$

$$
R^{2}=1-\frac{\sum_{j}\left\|\log \left(B S C_{\text {meas }}\left(k_{j}\right)\right)-\log \left(B S C_{\text {theo }}\left(k_{j}\right)\right)\right\|^{2}}{\sum_{j}\left\|\log \left(B S C_{\text {meas }}\left(k_{j}\right)\right)-\overline{\log \left(B S C_{\text {meas }}\right)}\right\|^{2}}
$$

where $B S C_{\text {theo }}$ represents the theoretical BSC computed with the estimated values of the

QUS parameters obtained by the minimization procedure, and $\overline{\log \left(B S C_{\text {meas }}\right)}$ represents the mean value of $\log \left(B S C_{\text {meas }}\right)$ over the studied frequency range. The BSC in log scale was used (instead of the BSC in linear scale) in order to give approximately the same weight to all frequencies. The closer the $R^{2}$ value is to 1 , the better is the fit of the model to the data. A negative value for $R^{2}$ means that a mere horizontal line passing through the average value of the $\log \left(B S C_{\text {meas }}\right)$ would fit data better than does the model.

\section{E. Optical images of flowing RBC aggregate in a plane-plane rheometer After}

the ultrasound measurements, a plane-plane rheometer (Anton Paar MCR 301, Saint Laurent, Canada) coupled with a light microscope (objective X20) was used to observe the flowing RBC aggregates (see Fig. 1). The blood sample was first sheared at $300 \mathrm{~s}^{-1}$ to disrupt RBC aggregates and then sheared at residual values of $3,6,11,22,45$ and $90 \mathrm{~s}^{-1}$, 
as performed previously in the US measurements. Because the RBCs are opaque to light, the optical measurements were only performed for the lower hematocrits of $10 \%$ and $20 \%$ and the rheometer gap was adjusted depending of the studied shear rates in order to observe the aggregate boundaries. The rheometer gap was equal to 100, 100, 80, 60, 40 and $20 \mu \mathrm{m}$, respectively for the shear rates $3,6,11,22,45$ and $90 \mathrm{~s}^{-1}$. Images were acquired using the optical microscope and a camera CCD and were segmented manually to obtain the aggregate contour. Segmentation consisted in identifying an aggregate and putting five points on its contour. The ellipse passing through these five points was traced and the disc radius having the equivalent ellipse area was calculated. When the level of aggregation is high, aggregates are superimposed on the optical images, and it becomes difficult to identify aggregate contours on a single image. In that case, aggregates were tracked on several consecutive optical images to facilitate contour delimitation (see Fig. 2). Around two hundred radii were registered for each studied shear rate and each hematocrit. Then, the histogram $h$ of the aggregate radius was fitted with a gamma distribution to estimate the mean aggregate size $\bar{r}_{\mathrm{opt}}^{*}$ and the distribution width $z_{\mathrm{opt}}^{*}$ by minimizing the cost function $\mathcal{F}^{\prime}$ defined as:

$$
\mathcal{F}^{\prime}\left(\bar{r}_{\mathrm{opt}}^{*}, z_{\mathrm{opt}}^{*}\right)=\sum_{i}\left\|h\left(r_{i}\right)-p_{\left(\bar{r}_{\mathrm{opt}}^{*}, z_{\mathrm{opt}}^{*}\right)}\left(r_{i}\right)\right\|^{2}
$$

We employed the routine fmincon of MATLAB, with the constraint $p_{\left(\bar{r}_{\mathrm{opt}}, z_{\mathrm{opt}}\right)}\left(r_{\mathrm{opt}}<a\right)=0$ (i.e., the aggregate radius should be larger than the $\mathrm{RBC}$ radius $a$ ), in order to be 
de Monchy et al., JASA, p. 18

consistent with the constraint that we adopted in the case of ultrasound signals.

\section{Results}

\section{A. Ultrasonic characterization of RBC aggregation Figure 3 represents a typical}

measured $B S C_{\text {meas }}$ for different shear rates at hematocrit $\phi=20 \%$, as well as the

corresponding fitted curves obtained with the monodisperse models (EMTSFM and SFSE), and the polydisperse EMTSFM. The QUS parameters were estimated from twelve measured $B S C_{\text {meas }}$ (corresponding to the twelve acquired B-mode images). The values of the goodness-of-fit statistic of each model to the experimental data were computed and are given in Table 1 for all the studied hematocrits. The goodness-of-fit statistic reveals that the monodisperse EMTSFM had the worst fit to the data, especially for shear rates smaller than $11 \mathrm{~s}^{-1}$ at hematocrits of 20 and $40 \%$. For all values of hematocrit, the polydisperse EMTSFM provided the best fit to the data, especially for the lower shear rates $3 \mathrm{~s}^{-1}$ and 6 $\mathrm{s}^{-1}$. The polydisperse EMTSFM gave overall satisfactory fitting curves with goodness-of-fit statistic comprised between 0.89 and 0.99 for the shear rates greater than $6 \mathrm{~s}^{-1}$, whatever the considered hematocrit (against goodness-of-fit statistics comprised between 0.49 and 0.99 for the SFSE model).

The QUS parameters estimated from the monodisperse and polydisperse EMTSFM are reported in Fig. 4 for hematocrit values of $\phi=10,20$ and 40\%. For the highest shear 
de Monchy et al., JASA, p. 19

rate of $90 \mathrm{~s}^{-1}$ (i.e. the smallest level of aggregation), the mean aggregate radius $\bar{r}_{\mathrm{ag}}^{*} / a$ obtained from the monodisperse and polydisperse EMTSFM is close to 1, corresponding to the case of disaggregated RBCs. The $B S C_{\text {meas }}$ amplitude, as well as the estimated aggregate radius $\bar{r}_{\mathrm{ag}}^{*} / a$, decreased when shear rate increased; i.e., when the level of aggregation became smaller, as expected. Typical examples of aggregate radius distribution obtained with the polydisperse EMTSFM are given in Figs. 4(a4), 4(b4) and 4(c4) for hematocrit values of $\phi=10 \%, 20 \%$ and $40 \%$. It is interesting to notice a decrease in the width of the aggregate radius PDF (as given by the standard deviation $\sigma_{\mathrm{ag}}^{*} / a$ ) when shear rate increases, since it is the first time that a QUS technique allows to provide the width distribution in the field of ultrasonic blood characterization.

Parameters $W^{*}$ and $D^{*}$ estimated with the SFSE model are reported in Fig. 5. Increasing the shear rates resulted overall in decreases of $W^{*}$ and $D^{*}$ (by excluding the shear rate $3 \mathrm{~s}^{-1}$ ). The SFSE could not follow the frequency dependence of the measured $B S C_{\text {meas }}$ at shear rates of 3 and $6 \mathrm{~s}^{-1}$ (i.e., for the higher level of aggregation), as reflected by drastically lower values of the goodness-of-fit statistic computed by Eq. (19) (see Table $1)$.

\section{B. Comparison of ultrasonic and optical aggregate measurements Aggregate}

size was also quantified using a plane-plane rheometer combined with an optical microscope (as described in section III.D) in order to evaluate the accuracy of the 
de Monchy et al., JASA, p. 20

aggregate size estimated by ultrasound. Figure 6 compares the aggregate radius PDF, the mean aggregate radius $\bar{r}_{\mathrm{opt}} / a$ and the standard deviation $\sigma_{\mathrm{opt}} / a$ estimated by optics and the corresponding values estimated by QUS (using the polydisperse EMTSFM) for hematocrit $\phi=10 \%$. Table 2 summarizes the correlation coefficients $r^{2}$ between mean aggregate radius estimated by optics and by ultrasound using the three scattering models, namely, the monodisperse SFSE and EMTSFM and the polydisperse EMTSFM. The mean aggregate radii estimated by the polydisperse EMTSFM agree well with those obtained from optical measurements, with correlation coefficients $0.90 \leq r^{2} \leq 0.98$ (see Table 2) and with a slope of linear regression around 1, for both studied hematocrit values of $10 \%$ and $20 \%$. Good correlation $\left(r^{2} \geq 0.80\right)$ was also found between the standard deviation estimated by optics and ultrasound. In the case of the optical data, where estimates of radii of aggregates were available, we have also estimated the corresponding gamma distributions without imposing the constraint that aggregates radii be no smaller than an RBC radius (see Eq. (20)). Whether this constraint was imposed or not for the optical data, similar correlations were obtained between ultrasonic and optical data.

\section{Discussion}

The goodness-of-fit values given in Table I and the fitting curves shown in Fig. 3 reveal that the polydisperse EMTSFM provided the best fit to data, whatever the shear rates and the hematocrit studied. However, for all three models, the smallest 
de Monchy et al., JASA, p. 21

goodness-of-fit values occurred at the lowest shear rate of $3 \mathrm{~s}^{-1}$. The polydisperse EMTSFM seems thus to reach its limit of applicability at the lowest shear rate of $3 \mathrm{~s}^{-1}$, corresponding to a large width distribution and a large aggregate size. Several assumptions made in the polydisperse EMTSFM might explain this limitation. The assumption of spherical aggregates used in the EMT theory might be less reliable for modeling a single RBC aggregate of variable shape at high frequencies. In the proposed in vitro experiments, the blood sample was prepared by using polymer dextran $500 \mathrm{kDa}$ to mimic pathological blood and to form clumps. The resulting aggregates have an ellipsoidal shape (see Fig. 2), rather than a spherical one as assumed by the EMT. Nevertheless, previous studies (see subsection V.D and Fig. 7 in Ref. (13)) demonstrated that the magnitude and frequency dependency of the form factor corresponding to randomly oriented ellipsoidal scatterers are in good agreement with those obtained from spherical ones of same volume within the range $k r_{\text {ag }}<1.5$. The major discrepancy in backscatter responses between randomly oriented ellipsoids and spheres of equivalent volume remains the magnitude of dips (see Fig. 7 in Ref. (13)). Moreover, the polydisperse EMTSFM assumed polydispersity in aggregate size, but not polydispersity in aggregate compactness or shape. Finally, the individual biconcave RBCs were also modeled as spheres of equivalent volume. Previous studies ${ }^{30 ; 31}$ demonstrated that biconcave RBC scattering differs by more than $5 \%$ from spheres of equivalent volume for frequencies above $20 \mathrm{MHz}$. The impact of this 
de Monchy et al., JASA, p. 22

simplification on the structural aggregate estimated in the 11-41 MHz bandwidth is unknown and still needs to be explored.

One can also notice that negative values of the goodness-of-fit statistic were obtained with the SFSE modeling for the lowest shear rate of $3 \mathrm{~s}^{-1}$. Moreover, the estimated aggregate diameter $D^{*}$ estimated by the SFSE did not agree with the expected tendency: the aggregate size is expected to increase while the shear rate decreases, whereas the estimated aggregate diameter $D^{*}$ decreased for the lowest shear rate of $3 \mathrm{~s}^{-1}$. This limitation of the SFSE for hyperaggregating blood was previously observed in experimental studies for $D^{*}>7.2$ corresponding to $k R^{*}>2\left(R^{*}\right.$ being the aggregate radius estimated by the SFSE). ${ }^{2}$ One can also notice the linear relationship between $W^{*}$ et $D^{*}$ in Fig. $5(\mathrm{c})$, as observed previously under experimental conditions ${ }^{1}$ and in 3-D numerical simulations. ${ }^{4 ; 5}$ This means that the BSC parameterization can be reduced to one parameter when using the SFSE.

Although the monodisperse EMTSFM did not produce a satisfactory goodness-of-fit statistic, correlation was found with the optical estimated radii, with $r^{2}$ superior to 0.85 . It is interesting to observe that both monodisperse and polydisperse EMTSFM gave similar values of the aggregate radius, and that the mean radii estimated with the monodisperse EMTSFM were slightly larger than those estimated with the polydisperse EMTSFM. For the highest shear rate of $90 \mathrm{~s}^{-1}$, the aggregate radius PDF estimated with the polydisperse 
de Monchy et al., JASA, p. 23

EMTSFM was quasi-monodisperse, and the monodisperse and polydisperse EMTSFM gave similar estimated radii, as expected. For shear rates less than $45 \mathrm{~s}^{-1}$ (i.e., for the higher levels of aggregation), the monodisperse EMTSFM provided radius estimates that fall within the radius range estimated with the polydisperse EMTSFM, as observed previously by Lavarello and Oelze ${ }^{32}$ when the effective scatterer size is estimated from a population of scatterers with a continuous size distribution. As an example, for the shear rate of $3 \mathrm{~s}^{-1}$, the polydisperse EMTSFM provided aggregate radii $r_{\mathrm{ag}} / a$ varying between 1 and 8 , with $\bar{r}_{\mathrm{ag}} / a=4$, for the S10 sample (and varying between 1 and 10, with $\bar{r}_{\mathrm{ag}} / a=5.2$, for the S40 sample), whereas the monodisperse EMTSFM estimated a normalized aggregate radius approximatively equal to 5 for the S10 sample (and approximatively equal to 8 for the S40 sample) (see Fig. 4).

When considering the polydisperse EMTSFM, the estimates $\bar{r}_{\mathrm{ag}}^{*} / a$ and $\sigma_{\mathrm{ag}}^{*} / a$ are similar for the two series of experiments and show low variance, whatever the studied hematocrit (see Fig. 4). Whereas, the estimates $\phi_{i}^{*}$ differ from the two series of experiments and show high variance (Figs. 4(a3) and 4(c3)). Netherveless, the aggregate compactness $\phi_{i}^{*}$ estimated with the polydisperse EMTSFM were found comprised between 0.8 and 1 , and seem to be in a reasonable range of values for deformable packed red blood cells.

To our knowledge, there is no known way to experimentally characterize the real size of RBC aggregates at a physiological hematocrit. That is why the optical measurements 
de Monchy et al., JASA, p. 24

were limited to hematocrits of $10 \%$ and $20 \%$. We observed a good agreement between optical and ultrasound measurements for the mean aggregate radius and the standard deviation of the aggregate radius distribution. However, the rheometer gap used in optics varies between 20 and $100 \mu \mathrm{m}$ (depending on the studied shear rate) in order to observe the aggregate boundaries. So, the set-up may suffer from wall effects, since the rheometer gap is about eight or nine times the mean aggregate radius. The gap should be around twenty times the mean aggregate radius to avoid wall effects. In comparison, the Couette gap of $2 \mathrm{~mm}$ used for ultrasonic experiments did not suffer from wall effects. This is why the comparison between ultrasonic and optical estimates is only qualitative. Netherveless, a good correlation was found between optics and ultrasound for the mean aggregate radius and distribution width, as reported in Table II and in Figure 6.

\section{Conclusion}

This study proposed a novel scattering ultrasound model for considering polydispersity in terms of aggregate size. Fitting the polydisperse model yielded aggregate size distributions that were consistent with direct light microscope observations at low hematocrits. It is interesting to notice that the width of the aggregate radius PDF estimated by the polydisperse EMTSFM is larger as the level of aggregation increases, whatever the studied hematocrit. This trend was observed not only on the QUS estimates but also on the optical observations. To our knowledge, it is the first experimental study 
de Monchy et al., JASA, p. 25

showing that an increase in the level of aggregation corresponds to an increase in the size distribution width. Further studies should be conducted on blood with different aggregation tendencies (promoted by different macromolecules, for instance) to confirm this observation.

Acknowledgment This research was supported by the French National Research Agency (Grant No. ANR-15-CE19-0017), and the Labex MEC (Grant No.

ANR-10-LABX-0092) and the A*MIDEX project (Grant No. ANR-11-IDEX-0001-02)

funded by the Investissements Avenir French Government program. Support was also obtained from the Canadian Institutes of Health Research (Grant \#MOP-84358).

\section{REFERENCES}

1. F. T. H. Yu and G. Cloutier, "Experimental ultrasound characterization of red blood cell aggregation using the structure factor size estimator", J. Acoust. Soc. Am. 122, 645-656 (2007).

2. E. Franceschini, F. T. H. Yu, F. Destrempes and G. Cloutier, "Ultrasound characterization of red blood cell aggregation with intervening attenuating tissue-mimicking phantoms", J. Acoust. Soc. Amer. 127, 1104-1115 (2010).

3. E. Franceschini, B. Metzger, and G. Cloutier, "Forward problem study of an effective 
de Monchy et al., JASA, p. 26

medium model for ultrasound blood characterization", IEEE Trans. Ultrason., Ferroelectr., Freq. Control 58, 2668-2679 (2011).

4. R. K. Saha, E. Franceschini, and G. Cloutier, "Assessment of accuracy of the structure-factor-size-estimator method in determining red blood cell aggregate size from ultrasound spectral backscatter coefficient," J. Acoust. Soc. Am. 129, 2269-2277 (2011).

5. E. Franceschini, R. K. Saha, and G. Cloutier, "Comparison of three scattering models for ultrasound blood characterization," IEEE Trans. Ultrason., Ferroelectr., Freq. Control 60, 2321-2334 (2013).

6. G. Barshtein, A. M. Ponizovsky, Y. Nechamkin, M. Ritsner, S. Yedgar, and L. D. Bergelson, "Aggregability of red blood cells of schizophrenia patients with negative syndrome is selectively enhanced", Schizophrenia Bulletin 30, 913 (2004).

7. P. A. Menichini, M. G. Larese, and B. D. Riquelme, "Automatic analysis of microscopic images of red blood cell aggregates, in SPIE Biophotonics South America, pp. 953132953132, International Society for Optics and Photonics (2015).

8. E. Franceschini, R. Guillermin, F. Tourniaire, S. Roffino, E. Lamy, and J.-F. Landrier, "Structure Factor Model for understanding the measured backscatter coefficients from concentrated cell pellet biophantoms", J. Acoust. Soc. Amer. 135, 3620-3631 (2014). 
de Monchy et al., JASA, p. 27

9. R. de Monchy, B. Chayer, G. Cloutier and E. Franceschini, "Effective Medium Theory combined with a polydisperse Structure Factor Model for characterizing red blood cell aggregation", in Proceedings of the IEEE Ultrasonics Symposium 2016.

10. A. Han and W. D. O'Brien, "Structure function for high-concentration biophantoms of polydiperse scatterer sizes", IEEE Trans. ultras. Ferroelectr. Freq. Control. 62, 303-318 (2015).

11. E. Franceschini, R. de Monchy and J. Mamou, "Quantitative characterization of tissue microstructure on concentrated cell pellet biophantoms based on the structure factor model", IEEE Trans. ultras. Ferroelectr. Freq. Control. 63, 1321-1334 (2016).

12. R. de Monchy, "Développement et évaluation d'une théorie de milieu effectif combinée à un facteur de structure polydisperse pour la caractérisation ultrasonore de l'agrégation érythrocytaire", PhD thesis, Aix-Marseille University (December 2016).

13. R. de Monchy, F. Destrempes, R. Saha, G. Cloutier and E. Franceschini, "Coherent and incoherent ultrasound backscatter from cell aggregates", J. Acoust. Soc. Am. 140, 2173-2184 (2016).

14. H. Schmid-Schonbein, G. Gallasch, J. V. Gosen, E. Volger, and H. J. Klose, "Red cell aggregation in blood flow. I. New methods of quantification", Klin. Wschr. 54, 149-157 (1976). 
de Monchy et al., JASA, p. 28

15. H. Schmid-Schonbein, H. Malotta, and F. Striesow, "Erythrocyte aggregation: causes, consequences and methods of assessment", Tijdschr NVKS 15, 88-97 (1990).

16. P. M. Morse and K. U. Ingard, "Theoretical Acoustics", (Princeton, NJ: Princeton University Press, 1968), Chap. 8, pp. 400-466.

17. M. F. Insana and D. G. Brown, "Acoustic scattering theory applied to soft biological tissues", in Ultrasonic Scattering in Biological Tissues, edited by K. K. Shung and G. A. Thieme (CRC, Boca Raton, FL, 1993), Chap. 4, pp. 76-124.

18. G. T. Kuster and M. N. Toksoz, "Velocity and attenuation of seismic waves in two-phase media: part I. Theoretical formulations", Geophysics 39, 587-606 (1974).

19. M. S. Wertheim, "Exact solution of the Percus-Yevick integral equation for hard spheres", Physical Review Letters 10, 321-323 (1963).

20. W. L. Griffith, R. Triolo, and A. L. Compere, "Analytical scattering function of a polydisperse Percus-Yevick fluid with Schulz distributed diameters", Phys. Rev. A 35, 2200-2206 (1987).

21. O. Baskurt, B. Neu, and H. J. Meiselman, "Mechanism of Red Blood Cell Aggregation", in Red blood cell aggregation (CRC Press, 2012), Chap.3, pp. 31-62.

22. R. Kuc and M. Schwartz, "Estimating the acoustic attenuation coefficient slope for 
de Monchy et al., JASA, p. 29

liver from reflected ultrasound signals", IEEE Trans. Sonics UltrasonicsSU-26, pp. 353-362 (1979).

23. L. C. Nguyen, F. T. H. Yu and G. Cloutier, "Cyclic changes in blood echogenicity under pulsatile flow are frequency dependent", Ultrasound in Med. \& Biol. 34, pp. 664-673 (2008).

24. L. X. Yao, J. A. Zagzebski and E. L. Madsen, "Backscatter coefficient measurements using a reference phantom to extract depth-dependent instrumentation factors", Ultrasonic Imaging 12, pp. 58-70 (1990).

25. S. H. Wang and K. K. Shung, "An approach for measuring ultrasonic backscattering from biological tissues with focused transducers", IEEE Trans. Biomed. Eng. 44, 549-554 (1997).

26. M. L. Oelze, and W. D. O'Brien, "Application of three scattering models to characterization of of solid tumors in mice", Ultrasonic Imaging 28, 83-96 (2006).

27. V. Twersky, "Low-frequency scattering by correlated distributions of randomly oriented particles", J. Acoust. Soc. Am. 81,1609-1618 (1987).

28. K. K. Shung, "On the ultrasound scattering from blood as a function of hematocrit", IEEE Trans. Sonics Ultrason. SU-29, 327-331 (1982). 
de Monchy et al., JASA, p. 30

29. M. L. Oelze, and W. D. O'Brien, "Application of three scattering models to characterization of of solid tumors in mice", Ultrasonic Imaging 28, 83-96 (2006).

30. D. Savery and G. Cloutier, "High-frequency ultrasound backscattering by blood: Analytical and semi-analytical models of the erythrocyte cross section", J. Acoust. Soc. Am., vol. 23, no. 4, pp. 3963-3971, 2007.

31. C. C. Coussios, "The significance of shape and orientation in single-particle weak-scatterer models", J. Acoust. Soc. Am., vol. 112, pp. 906-915, 2002.

32. R. Lavarello, and M. Oelze, "Quantitative ultrasound estimates from populations of scatterers with continuous size distributions : Effects of the size estimator algorithm", IEEE Trans. Ultras. Ferroelectr. Freq. Control. 59, 2066-2076 (2012).

33. https://blogs.mathworks.com/steve/2006/06/02/cell-segmentation/

34. Y. Chen, K. Biddell, A. Sun, P. A. Relue and J. D. Johnson, "An automatic cell counting method for optical images", Proc. of the first joint BMES/EMBS conference (1999). 
de Monchy et al., JASA, p. 31

\section{TABLE CAPTIONS}

Table I. Goodness-of-fit statistic (averaged over the twelve BSC measurements) of the polydisperse EMTSFM (EMT poly $\left._{1}\right)$, the monodisperse EMTSFM (EMT mono $)$ and the SFSE modeling for the blood samples sheared at several shear rates and for hematocrits of 10, 20 and 40\% (denoted S10, S20 and S40).

Table II. Correlation coefficient $r^{2}$ between mean aggregate radius estimated by optics and by ultrasound (using the three scattering models). 
de Monchy et al., JASA, p. 32

Table 1: Goodness-of-fit statistic (averaged over the twelve BSC measurements) of the polydisperse EMTSFM $\left(\mathrm{EMT}_{\text {poly }}\right)$, the monodisperse EMTSFM (EMT $\left.\mathrm{E}_{\text {mono }}\right)$ and the SFSE modeling for the blood samples sheared at several shear rates (and for hematocrits 10, 20 and $40 \%$ (denoted S10, S20 and S40) .

\begin{tabular}{cccccccc}
\hline Shear & rate $\left(\mathrm{s}^{-1}\right)$ & 3 & 6 & 11 & 22 & 45 & 90 \\
\hline \multirow{3}{*}{$\mathrm{S} 10$} & EMT $_{\text {poly }}$ & 0.77 & 0.90 & 0.97 & 0.97 & 0.98 & 0.98 \\
& EMT $_{\text {mono }}$ & -0.44 & 0.68 & 0.96 & 0.96 & 0.97 & 0.98 \\
& SFSE $^{*}$ & -1.11 & 0.49 & 0.97 & 0.97 & 0.98 & 0.97 \\
\hline \multirow{2}{*}{$\mathrm{S} 20$} & EMT $_{\text {poly }}$ & 0.69 & 0.89 & 0.97 & 0.98 & 0.98 & 0.97 \\
& EMT $_{\text {mono }}$ & -13.33 & 0.52 & 0.93 & 0.96 & 0.98 & 0.97 \\
& SFSE & -1.18 & 0.62 & 0.97 & 0.96 & 0.96 & 0.97 \\
\hline \multirow{2}{*}{$\mathrm{S} 40$} & EMT $_{\text {poly }}$ & 0.44 & 0.96 & 0.99 & 0.98 & 0.99 & 0.99 \\
& $\mathrm{EMT}_{\text {mono }}$ & -25.23 & -2.00 & -0.02 & 0.95 & 0.99 & 0.99 \\
& $\mathrm{SFSE}$ & -2.68 & 0.77 & 0.95 & 0.94 & 0.99 & 0.99 \\
\hline
\end{tabular}


de Monchy et al., JASA, p. 33

Table 2: Correlation coefficient $r^{2}$ between mean aggregate radius estimated by optics and by ultrasound (using the three scattering models).

\begin{tabular}{cccc}
\hline & SFSE & EMT $_{\text {mono }}$ & EMT $_{\text {poly }}$ \\
\hline S10 - Series 1 & 0.75 & 0.85 & 0.94 \\
\hline S10 - Series 2 & 0.84 & 0.92 & 0.98 \\
\hline S20 - Series 1 & 0.82 & 0.95 & 0.92 \\
\hline S20 - Series 2 & 0.76 & 0.85 & 0.90 \\
\hline
\end{tabular}


de Monchy et al., JASA, p. 34

\section{FIGURE CAPTIONS}

Figure 1. (Color online) Schematic illustration of the plane-plane rheometer combined with optical microscope seen on the sideway view.

Figure 2. (Color online) (On the left) Consecutive optical images in the plane-plane rheometer coupled with a light microscope for a shear rate of $6 \mathrm{~s}^{-1}$ at a hematocrit of 10\%. One can distinguish the displacement of an aggregate surrounded with dotted lines. When the aggregate can be separated from the others, the aggregate contour is outlined (see red solid line). (On the right) Example of radius histogram fitted with a gamma distribution.

Figure 3. (Color online) Examples of measured $B S C_{\text {meas }}$ at different shear rates with hematocrit $\phi=20 \%$ (symbols). (a) Corresponding fitted curves obtained with the monodisperse SFSE (dotted lines) and monodisperse EMTSFM (dashed lines). (b) Corresponding fitted curves obtained with the polydisperse EMTSFM (solid lines).

Figure 4. (Color online) (a) Values of $\bar{r}_{\text {ag }}^{*} / a, \sigma_{\mathrm{ag}}^{*} / a$ and $\phi_{i}^{*}$ estimated by the polydisperse EMTSFM $\left(\mathrm{EMT}_{\text {poly }}\right)$ and by the monodisperse EMTSFM (EMT mono $)$ at different shear rates for the two series of experiments and for a hematocrit $\phi=10 \%$. Also represented are the corresponding aggregate radius probability distribution function (PDF) estimated by the polydisperse EMTSFM for one series. (b) Same as 
(a) for a hematocrit $\phi=20 \%$. (c) Same as (a) for a hematocrit $\phi=40 \%$.

Figure 5. (Color online) (a) Packing factor $W^{*}$ and (b) aggregate diameter $D^{*}$ estimated with the SFSE model at different shear rates for the two series of experiments and for hematocrit values of $\phi=10,20$ and $40 \%$. (c) Linear relations between $W^{*}$ and $D^{*}$ for hematocrit values of $\phi=10,20$ and $40 \%$.

Figure 6. (Color online) (a) Comparison of aggregate radius PDF estimated by US (solid line) and optics (dashed line) at a hematocrit of $\phi=10 \%$. (b) Comparison of mean aggregate radius $\bar{r}_{\mathrm{ag}}^{*} / a$ estimated by the polydisperse SFM with mean aggregate radius obtained by optics for the two series of experiments. (c) Comparison of standard deviation of aggregate radius PDF $\sigma_{\mathrm{ag}}^{*} / a$ estimated by the polydisperse SFM with standard deviation obtained by optics for the two series of experiments. 
de Monchy et al., JASA, p. 36

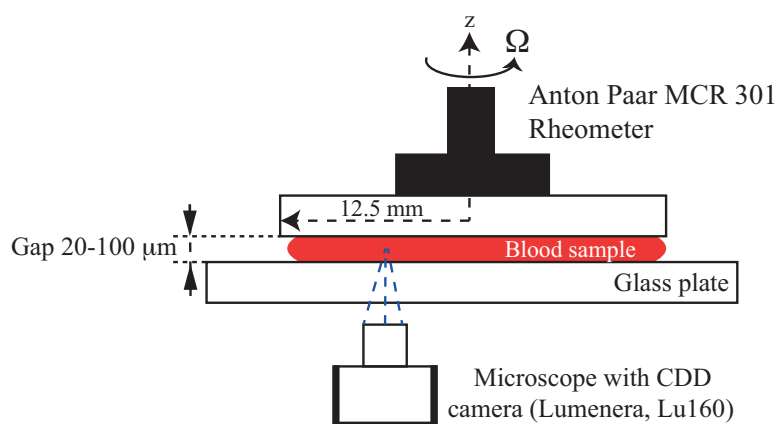

Figure 1: (Color online) Schematic illustration of the plane-plane rheometer combined with optical microscope seen on the sideway view. 
de Monchy et al., JASA, p. 37
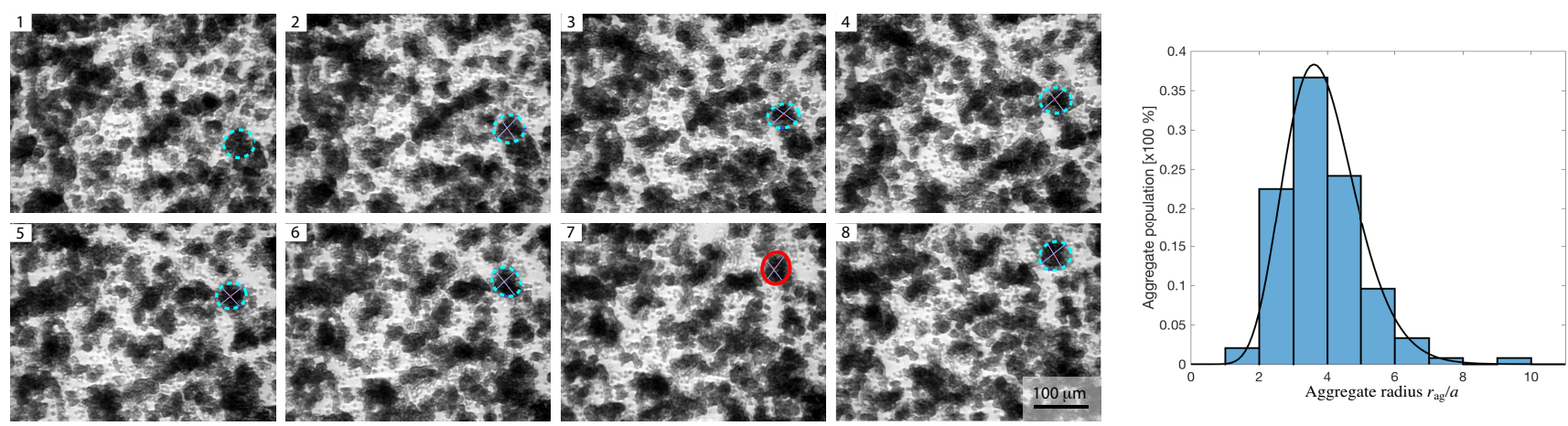

Figure 2: (Color online) (On the left) Consecutive optical images in the plane-plane rheometer coupled with a light microscope for a shear rate of $6 \mathrm{~s}^{-1}$ at a hematocrit of $10 \%$. One can distinguish the displacement of an aggregate surrounded with dotted lines. When the aggregate can be separated from the others, the aggregate contour is outlined (see red solid line). (On the right) Example of radius histogram fitted with a gamma distribution. 
de Monchy et al., JASA, p. 38
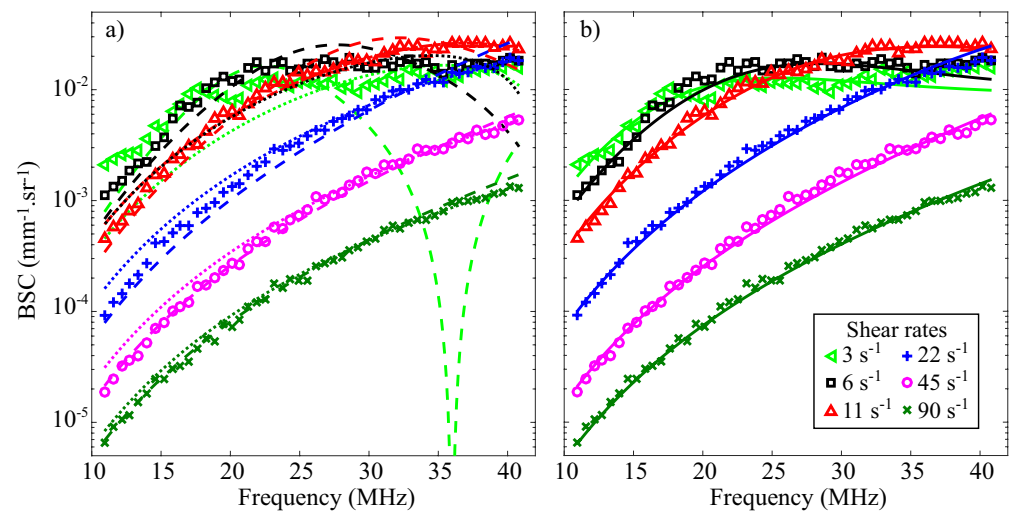

Figure 3: (Color online) Examples of measured $B S C_{\text {meas }}$ at different shear rates with hematocrit $\phi=20 \%$ (symbols). (a) Corresponding fitted curves obtained with the monodisperse SFSE (dotted lines) and monodisperse EMTSFM (dashed lines). (b) Corresponding fitted curves obtained with the polydisperse EMTSFM (solid lines). 

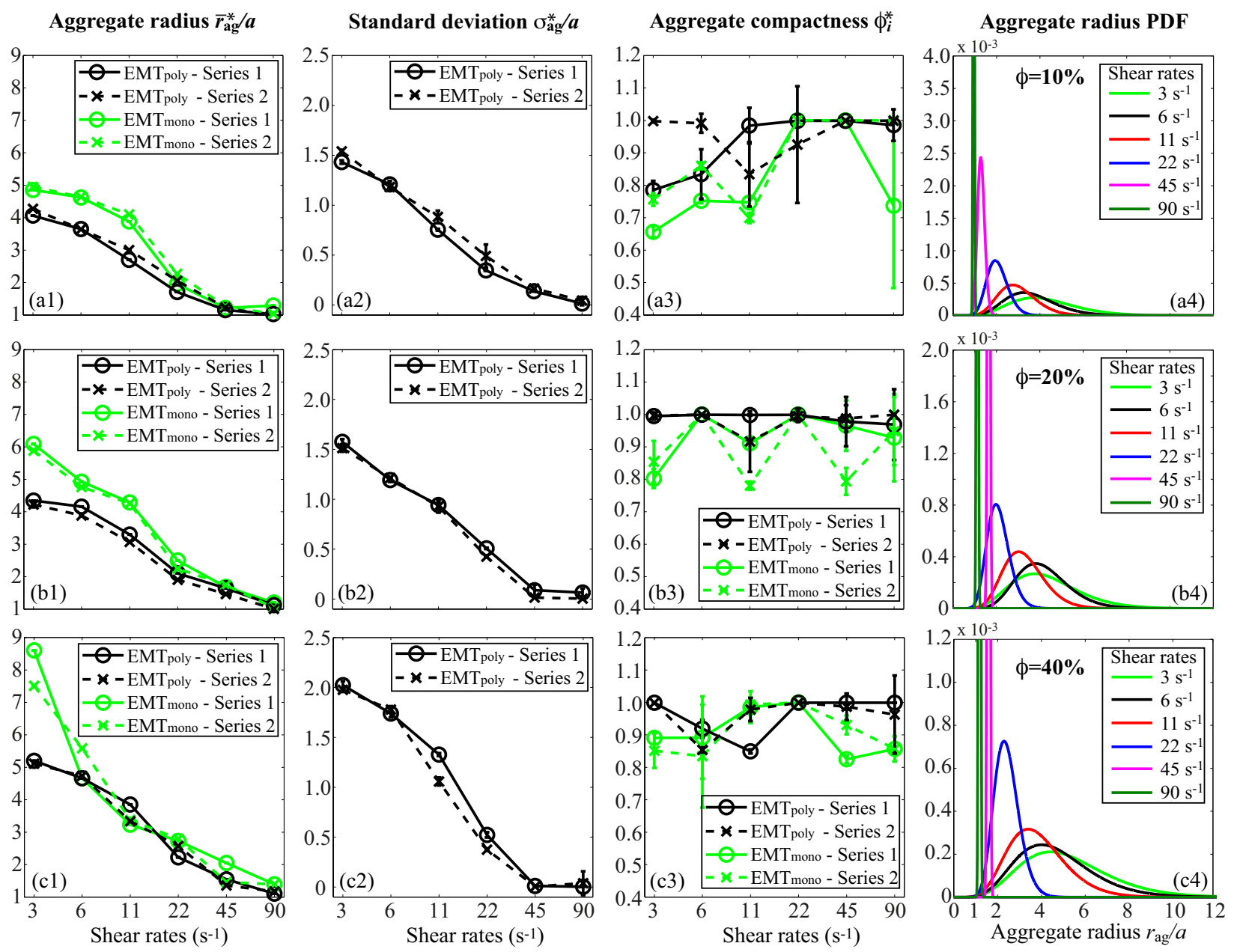

Figure 4: (Color online) (a) Values of $\bar{r}_{\mathrm{ag}}^{*} / a, \sigma_{\mathrm{ag}}^{*} / a$ and $\phi_{i}^{*}$ estimated by the polydisperse EMTSFM $\left(\mathrm{EMT}_{\text {poly }}\right)$ and by the monodisperse EMTSFM $\left(\mathrm{EMT}_{\text {mono }}\right)$ at different shear rates for the two series of experiments and for a hematocrit $\phi=10 \%$. Also represented are the corresponding aggregate radius probability distribution function (PDF) estimated by the polydisperse EMTSFM for one series. (b) Same as (a) for a hematocrit $\phi=20 \%$. (c) Same as (a) for a hematocrit $\phi=40 \%$. 
(a)

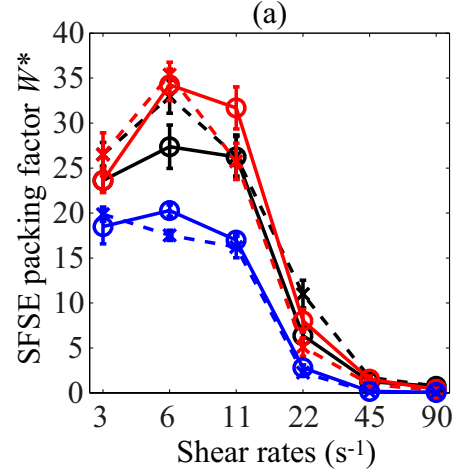

(b)

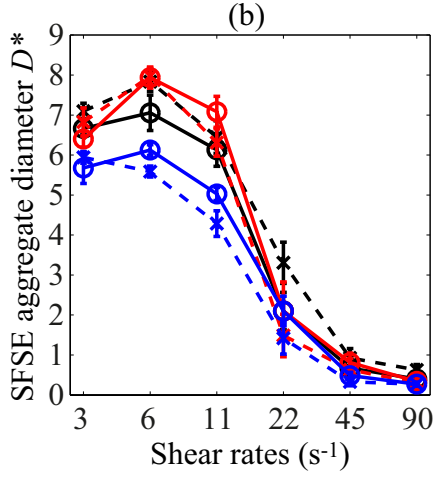

(c)

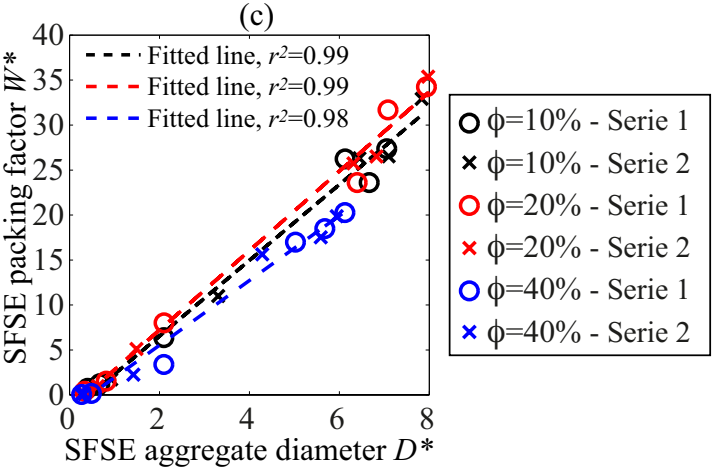

Figure 5: (Color online) (a) Packing factor $W^{*}$ and (b) aggregate diameter $D^{*}$ estimated with the SFSE model at different shear rates for the two series of experiments and for hematocrit values of $\phi=10,20$ and $40 \%$. (c) Linear relations between $W^{*}$ and $D^{*}$ for hematocrit values of $\phi=10,20$ and $40 \%$. 

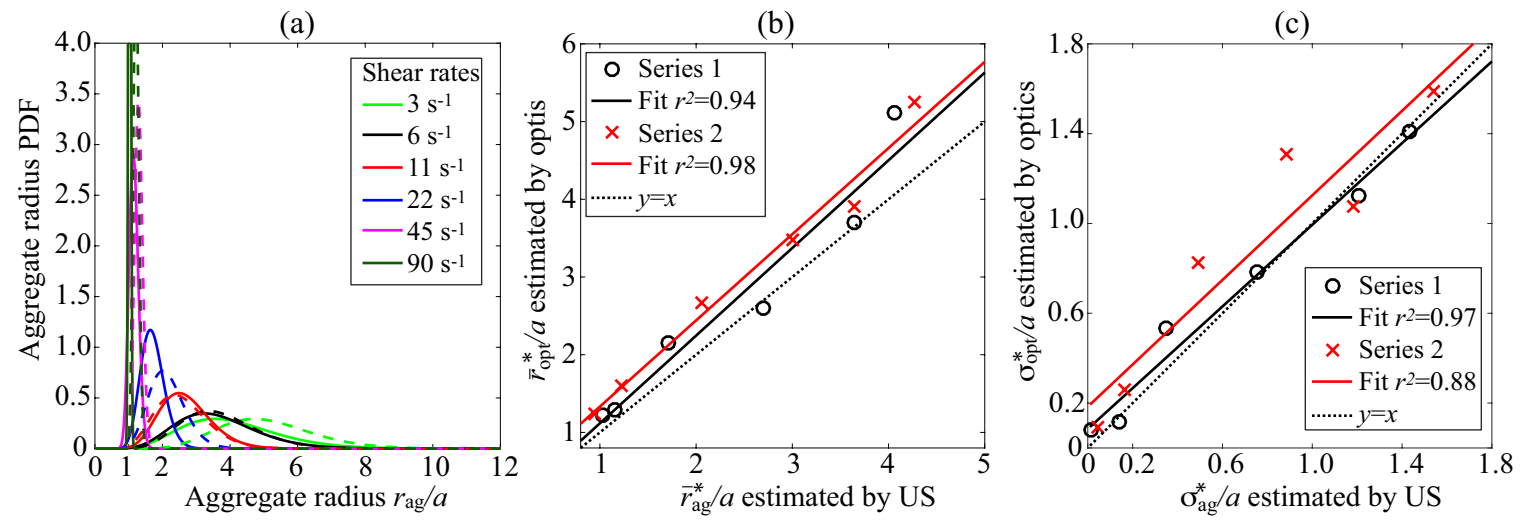

Figure 6: (Color online) (a) Comparison of aggregate radius PDF estimated by US (solid line) and optics (dashed line) at a hematocrit of $\phi=10 \%$. (b) Comparison of mean aggregate radius $\bar{r}_{\text {ag }}^{*} / a$ estimated by the polydisperse SFM with mean aggregate radius obtained by optics for the two series of experiments. (c) Comparison of standard deviation of aggregate radius $\mathrm{PDF} \sigma_{\mathrm{ag}}^{*} / a$ estimated by the polydisperse SFM with standard deviation obtained by optics for the two series of experiments. 